\title{
Refractory Abdominal Neuroendocrine Neoplasm
}

National Cancer Institute

\section{Source}

National Cancer Institute. Refractory Abdominal Neuroendocrine Neoplasm. NCI

Thesaurus. Code C138184.

An abdominal neuroendocrine neoplasm that does not respond to treatment. 